\title{
Lidil
}

Revue de linguistique et de didactique des langues

\section{Lecture littéraire et disqualification scolaire}

\section{Bertrand Daunay}

\section{OpenEdition}

\section{Journals}

Édition électronique

URL : http://journals.openedition.org/lidil/51

DOI : $10.4000 /$ lidil.51

ISSN : 1960-6052

\section{Éditeur}

UGA Éditions/Université Grenoble Alpes

\section{Édition imprimée}

Date de publication : 1 juin 2006

Pagination : 19-36

ISBN : 2-914176-14-7

ISSN : $1146-6480$

Référence électronique

Bertrand Daunay, «Lecture littéraire et disqualification scolaire », Lidil [En ligne], 33 | 2006, mis en ligne le 05 décembre 2007, consulté le 19 avril 2019. URL : http://journals.openedition.org/lidil/51 ; DOI 10.4000/lidil.51

Ce document a été généré automatiquement le 19 avril 2019

(C) Lidil 


\title{
Lecture littéraire et disqualification scolaire
}

\author{
Bertrand Daunay
}

1 Pourquoi veulent-ils tuer le français? Tel est le titre de l'ouvrage récent de Bernard Lecherbonnier (2005), qui ne craint pas d'user des procédés rhétoriques les plus éculés, dans ses moyens d'expression comme dans sa thématique'. Puisant à la veine conspirationniste du pamphlet', il veut dénoncer le fruit des agissements de la « cinquième colonne » - titre du premier chapitre - ou l'effet de la « trahison des clercs »

- titre du deuxième chapitre ${ }^{3}$. Dans celui-ci, l'auteur s'en prend à Alain Viala (l'un des concepteurs principaux des derniers programmes de français pour le lycée) et donne à juger de la rigueur de sa propre pensée par cette envolée à relents révisionnistes :

L'objectif d'Alain Viala est clair : émasculer la littérature, en faire une catégorie de la communication, supprimer toute référence à la notion de style, évacuer toute idée d'esthétique. Aucun régime totalitaire n'a eu l'audace de pousser la censure jusqu'à cette limite. On a vu des régimes expurger la tradition littéraire de leur pays, expulser des écrivains à cause de leur origine ethnique ou religieuse - ainsi des nazis condamnant les écrivains juifs à l'oubli -, à cause de leurs opinions politiques ou de leurs convictions morales, mais on n'a jamais vu, à l'instar des talibans, des censeurs à ce point altérés de haine contre l'humanisme condamner la littérature au motif qu'elle est littéraire, pour ce qu'elle contient de littérature.

2 Une telle citation ne vaudrait pas d'être reproduite si elle n'était la dernière expression en date (à ce jour et à ma connaissance) d'un discours qui s'est déchainé au début de ce siècle suite à la parution des derniers programmes de français pour le lycée. Et surtout, ces mots illustrent la perte de tout repère rationnel qui caractérise (comme je tenterai de le montrer) certains discours de « défense » de la langue ou de la littérature - unies dans un même destin, puisque, si l'on en croit par exemple l'Académie française (2000), « aujourd'hui, la langue et la littérature elles-mêmes ainsi que les exercices qui en sont les instruments d'étude semblent être devenues les ennemis à détruire ».

Ces propos ressortissent aux avatars de la crise-du-français, dont le numéro 118 de ÉLA analyse le discours. Pierre Boutan et Dan Savatovsky (2000: 134), dans leur avant-propos à ce numéro, situent sa première manifestation entre 1906 et 1914, période où la formule 
«la crise du français », nouvelle, se développe et se diffuse rapidement, suite à des articles ou des ouvrages polémiques. Ils écrivent :

Nous proposons d'admettre que la première crise-du-français constitue en France et parfois dans d'autres pays francophones [...] la matrice d'une série de «crises» du même genre, de répliques ou de rechutes plus ou moins affaiblies, à la portée, à la durée, à la dramaturgie, au contexte sans doute différents, mais qui paraissent comme autant de jalons dans une histoire du français identifiée à ses moments critiques 4 .

4 Il y a en effet une matrice du discours de crise, qui rend si familiers les pamphlets divers qui sont publiés, à intervalles réguliers, sur le français - la langue comme son enseignement. Mais on peut généraliser le propos et l'élargir à certains discours intercrise, si je puis dire, qui sont comme engendrés par cette même matrice. C'est ce qui explique que l'on puisse trouver, tout au long du xxe siècle et jusqu'à nos jours les mêmes mots, le même ton, les mêmes intentions quand il s'agit de parler du français à l'école. Je chercherai à le montrer en me restreignant aux discours tenus sur ce que leurs auteurs considèrent eux-mêmes comme le cœur de l'enseignement du français : la littérature.

Plus précisément encore, je voudrais montrer combien il est aisé, quand il s'agit de l'enseignement de la littérature, de laisser s'épanouir un discours de disqualification". C'est particulièrement vrai, on le verra, quand il s'agit de la lecture littéraire, qui autorise souvent, à partir de présupposés non interrogés, un discours d'exclusion de pratiques jugées non légitimes.

6 Une dernière précision liminaire : il va de soi que, parcourant un siècle de discours, je citerai des auteurs fort différents ayant écrit parfois dans des contextes qui n'ont que peu de choses en commun : par exemple, pour prendre deux contemporains, Lanson n'est pas Agathon : ils se combattent même, et mes affinités avec le premier comme mon peu de sympathie pour le second m'interdisent de les confondre; pour prendre un autre exemple, Servais Étienne et Alain Finkielkraut, à un demi-siècle de distance, n'ont ni les mêmes projets ni les mêmes conceptions de l'enseignement et de la littérature. Mais, comme le disaient Pierre Boutan et Dan Savatovsky (2000: 134), si l'on traque la permanence d'un discours (en l'occurrence sur la littérature ou la lecture à l'école), c'est précisément pour faire ressortir certains traits structurels - mutatis mutandis.

Disqualification sociale

Dans les pamphlets du début du xxe siècle, les charges sont nombreuses contre les tenants de la démocratisation de l'enseignement secondaire, à une époque où l'opposition primaire/secondaire était celle de deux ordres de scolarisation, socialement discriminés. Dans ces querelles, ce qui est en cause est la sauvegarde de l'enseignement classique, caractérisé par les langues anciennes et la littérature. Dans les années 1910, eut lieu une vaste querelle contre la Sorbonne, menée par le parti intellectuel d'extrême droite, avec des attaques virulentes contre Lanson, la Sorbonne en général et les pédagogues en particulier - où l'on trouve, comme on verra, la rhétorique des opposants aux derniers programmes de français au lycée...

8 Voici par exemple ce que pouvait dire un certain André Beaunier, romancier et critique (1912: 100 sq.) :

Pour que le triomphe des primaires devînt plus commode, et pour ne pas avoir à les surmener, on a imaginé ce stratagème: avilir et, peu à peu, supprimer tout enseignement supérieur à celui que les primaires sont capables de recevoir. Et, avec une industrieuse hypocrisie, on s'est mis à la besogne. On n'a pas officiellement 
annoncé qu'on supprimait l'enseignement secondaire. Mais on l'a saccagé. Pour en finir assez rondement avec lui, on l'a touché au cœur, quand on a dénigré, quand on a réduit à un minimum ridicule et quand on a traité comme un vain accessoire toute la partie véritablement « classique » de ses programmes. secondaire, voire le supérieur, dans le domaine essentiel de l'époque, à savoir les études classiques. On voit bien le caractère socialement discriminant : les paysans n'ont pas leur place au lycée. Or cette idée se retrouve explicitement énoncée plus tard (1933), ailleurs (en Belgique) et sans aucune intention polémique (il s'agit de décrire une méthode d'explication de texte), chez Servais Étienne qui, dans un article de 1933, après avoir noté «l'embarras » des élèves devant les règles de l'explication de texte, ajoutait (p. 164) : « Un certain nombre se découragent, et c'est tant mieux : Mascarille a d'autres occupations qui l'attendent dans le vaste monde. » François Desonay explicite ce propos dans le portraitsouvenir qu'il consacre à Servais Étienne : « Il n'hésitait pas à rappeler aux médiocres que l'agriculture manque de bras » (Étienne, 1965 : 22).

On dira que ce sont là des propos d'une époque révolue. Il est vrai qu'un tel racisme social ne se trouve plus aussi répandu de nos jours, sauf quand le propos, sous les plumes les plus sérieuses, dérape - précisément à propos de littérature. Ainsi, Michel Picard (une référence obligée des didacticiens de la « lecture littéraire ») peut écrire sans fard (1986: 242) :

Il faut le dire brutalement, au risque d'effaroucher ou de blesser les belles âmes de tout bord dans leur rêve ingénument démagogique d'apporter la culture au peuple, 
instinctivement réceptif: l'effet littérature n'est concevable que pour le joueur expérimenté, l'« amateur » averti. On peut, on doit déplorer le caractère élitiste et sélectif de la chose - non le nier sottement. je disais voir la même rhétorique que dans les libelles cités plus haut : il s'agit d'un texte collectif, «C'est la littérature qu'on assassine rue de Grenelle », paru dans Le Monde du 4 mars 2000. Là, les auteurs, au contraire, dans leur rejet d'une réforme qui « assassinerait » la littérature, s'intéressent au peuple... Voyons dans quels termes :

N'alléguons pas Péguy, dont la mère rempaillait des chaises, qui entra rue d'Ulm avant de devenir écrivain, et qui sans la formation de cette école-là ne fût pas songeons-y un instant - devenu écrivain, ni Camus, aussi pauvre et aussi démuni et qui doit se retourner dans sa tombe, ni tant d'autres encore. Mais enfin, c'est de cela qu'il s'agit. Les petits Péguy d'aujourd'hui sont bien souvent des beurs : quelle plus grande preuve d'estime, d'intérêt et de respect pourrions-nous leur manifester que de leur donner la chance de la culture, au lieu de leur claquer la porte au nez en décrétant: "Pas de littérature pour vous!» Quelle meilleure chance d'intégration donner aux jeunes immigrés que de leur apprendre le latin et le grec pour qu'ils ouvrent les yeux sur l'unité de cette culture méditerranéenne qui est à la fois la leur et la nôtre?

17 Les beurs (ou les pauvres) et nous: on voit bien le sens que donnent les auteurs à ce qu'ils appellent «l'intégration ». Lesquels supposent que les «beurs », a priori, n'ont pas de culture et que seule celle que nous leur apportons peut leur être bénéfique ; le latin et le grec, évidemment - et la littérature classique (c'est l'objet du pamphlet)... C'est la même rhétorique que dans le libelle de l'Académie française (2000):

L'Académie rappelle à nouveau que l'école républicaine a été conçue en vue de corriger les effets des inégalités d'origine sociale et non en vue de les accentuer. C'est désespérer de l'école que de la déclarer, dans son principe, incapable d'initier les enfants et les adolescents d'origines diverses au plaisir de comprendre nos chefs-d'œuvre littéraires. Ceux qui ne trouvent pas, dans leur milieu, d'incitation suffisante à découvrir ces chefs-d'œuvre, ni même l'occasion d'en entendre parler, n'est-ce pas de l'école qu'ils sont en droit d'attendre ce que la famille n'a pu leur donner? Renoncer à faire jouer ce rôle correcteur à l'école et affaiblir, sous ce prétexte fallacieux, l'enseignement littéraire pour tous, n'est-ce pas œuvrer au contraire de ce que l'on prétend rechercher, puisque dès lors, la démission de l'école consacre les inégalités culturelles d'origine sociale?

$\mathrm{Au}$ fait : quand s'expriment les défenseurs de la littérature de l'Académie française ou de Sauver les lettres (collectif soutenu par certains des signataires du pamphlet cité plus haut), ce n'est que pour parler du secondaire « ordinaire » : le collège et le lycée - unis par l'histoire, les concours de recrutement, les corps d'inspection et les représentations dominantes dans le monde de l'éducation. A-t-on connu une polémique qui soit née de l'inquiétude de l'Académie française quant aux études littéraires en lycée professionnel ? Ou à propos des jeunes sortis du système scolaire avant d'avoir accédé au lycée?

L'assassinat de la littérature, que pourfendent ces auteurs, n'est en effet jamais que celui d'une certaine conception de la littérature et de son enseignement, circonscrite à celle qui dominait au cours de leurs propres études secondaires et universitaires et qui n'avait cours que dans les lieux qu'ils ont fréquentés...

Et c'est ce qui montre bien que le discours méprisant (Agathon), condescendant (Picard), compassionnel (nos modernes) sont des formes différentes d'un même discours de négation de l'altérité, au nom de valeurs culturelles jamais interrogées. Dans tous les cas, les auteurs décrètent la littérature étrangère à certaines classes sociales, pour se 
scandaliser soit que ces dernières accèdent au niveau d'apprentissage qui l'enseigne, soit que l'on pose la question de l'adéquation de son enseignement à ces élèves.

Disqualification scolaire

21 Si les didacticiens et les pédagogues (ces bêtes noires des opposants à la dernière réforme du lycée $e^{8}$ ) ont un mérite, ce n'est pas dans les solutions qu'ils proposent (toujours contestables et discutables), c'est dans le fait de ne pas éluder la question centrale de l'enseignement de la littérature (comme de la langue) : les pratiques langagières et culturelles, en tant qu'elles ne sont pas socialement neutres, nécessitent un enseignement-apprentissage qui prenne en compte les pratiques des élèves, notamment leur rapport à la culture littéraire et au traitement scolaire de cette culture.

C'est à peu près ce que disait le premier ennemi des Agathon, Gustave Lanson, en d'autres termes que les nôtres aujourd'hui $(1909: 3)$ :

Le mal consiste en ce que nous donnons à nos élèves, de moins en moins aptes à le recevoir, un enseignement de moins en moins propre à leur être communiqué. Nous avons aujourd'hui des élèves dont le plus grand nombre nous arrivent de leurs familles mal préparées à étudier de manière littéraire la langue et la littérature françaises.

23 À cette époque où l'inflation atteint (déjà) les effectifs du lycéé, il est amené à se demander (1909: 19) :

Ne faudrait-il pas opter entre l'enseignement et les élèves? Voici ce que je veux dire: si nous estimons que l'enseignement $d u$ français doive consister dans la communication d'une culture raffinée, d'une délicatesse extrême de goût, dans l'apprentissage des jouissances les plus fines de la littérature, que ce soit là l'essentiel de notre tâche, alors il nous faut nous demander, - en criant le plus haut possible et en nous associant, puisque c'est le bon moyen, - que cet enseignement, qui n'est bon en tout cas que pour une élite, nous ne le donnions qu'à une élite. Il faut renoncer aux neuf dixièmes de nos élèves; il faut demander qu'on transforme les collèges et les lycées en établissements primaires supérieurs [...].

Ou bien acceptons l'idée que l'enseignement secondaire est fait pour être donné à un très grand nombre d'élèves [...]. Mais alors il faut adapter notre enseignement à cette clientèle.

24 La position de Gustave Lanson est connue: il considère que la massification de l'enseignement (il parle lui-même des masses) est un fait irréversible, qu'il s'agit de prendre en compte en modifiant les pratiques et les objectifs de l'enseignement. Ce qui frappe dans ces mots de Lanson est la reconnaissance (au début du xxe siècle) d'un fait sociologique structurel (l'élargissement constant du public lycéen) et d'une nécessité didactique constante (la nécessaire adaptation de l'enseignement au nouveau public) objets d'une dénégation non moins constante et structurelle des discours conservateurs sur l'enseignement littéraire.

25 À son époque déjà, le même Gustave Lanson mettait en cause les objets d'enseignement (dont... la dissertation, en 1903) mais surtout la séparation des deux ordres du primaire et du secondaire (1905). De fait, la question de l'unification du système scolaire est une autre thématique permanente des discours sur l'enseignement : il faut se demander d'ailleurs si l'on ne retrouve pas le sourd écho du discours de ségrégation entre primaire et secondaire, dont on a vu plus haut quelques réalisations, dans les propos actuels sur l'opposition entre collège et lycée, certains semblant regretter qu'il n'y ait plus là deux ordres d'enseignement mais seulement deux niveaux, depuis l'unification du collège. C'est le cas dans les débats récents sur l'enseignement du français, dont deux traits méritent à 
cet égard d'être signalés : d'une part l'établissement d'une hiérarchie entre les deux composantes principales de la discipline - la langue et la littérature - et leur distribution selon les niveaux d'enseignement ( $c f$. par exemple Zink, 2000: 53 sq.); d'autre part le discrédit jeté sur l'écriture d'invention, instituée par les derniers programmes comme une des formes possibles d'accès à la littérature - et à la lecture littéraire, du seul fait qu'elle est supposée propre au collège ( $c f$. par exemple Jarrety, $2000: 9)^{10}$.

Ces discours" établissent une frontière entre les niveaux d'enseignement et les pratiques différenciées de la langue et de la littérature qui leur seraient propres ${ }^{12}$. Une opposition entre collège et lycée dans l'approche de la langue et de la littérature pourrait n'avoir rien de choquant, s'il ne s'agissait que d'envisager la progressivité des apprentissages. Mais ce qui est en jeu dans ces discours est bien différent : un trait récurrent dans les discours de "défense » de la littérature est précisément la négation de la progressivité des apprentissages, par l'institution d'une coupure, selon les niveaux, entre deux types de rapports au texte littéraire, qui seraient irréductibles ${ }^{13}$.

Disqualification des lectures et des lecteurs

C'est cet aspect que je voudrais aborder maintenant, en centrant mon approche sur certains discours sur la lecture, pour montrer comment ressurgissent des hiérarchies posées comme naturelles, disqualifiant certains rapports à la lecture qui ne correspondent pas à celui qui est seul conçu comme légitime ${ }^{14}$.

Gustave Lanson, dont a vu pourtant qu'il mettait à mal les préjugés scolaires de son époque, nous donnera un exemple assez parlant de cette structure de discours, à propos de l'explication de texte $(1925: 39)$ :

Par l'explication, un professeur de lycée ou d'université se propose d'apprendre à lire à ses élèves. L'instituteur apprend à lire l'alphabet, et le professeur de lycée ou d'université apprend à lire la littérature [...] Quand il s'agit de textes littéraires [...] il ne suffit pas, pour les bien lire, d'avoir appris à lire à l'école.

Étrange usage de la langue qui donne au même mot deux sens (que semble simplement différencier la typographie), selon une conception préalable de la lecture légitime - qui trouve à s'épanouir à propos de la littérature.

On sort de la première guerre du siècle ; sortons de la suivante pour lire, sous la plume de Jean Guéhenno, une même dichotomie et un même usage différencié du mot lire (1954: 25) « la grande masse des hommes n'a surement jamais tant lu. Mais il y a lire et lire» (p. 25). «Il faudrait justement lui apprendre à lire, à être seul avec un livre, le reconduire à sa solitude et la lui faire aimer. Ainsi préparerait-on des liseurs » (p. 25).

31 Le « vrai » lecteur gagne ici un nom : le liseur. Albert Thibaudet en avait dessiné la figure quelques années plus tôt; voulant distinguer le liseur de roman ("qui indique une habitude et un goût ») du lecteur (« qui peut impliquer un simple contact accidentel»), il écrit (1925 : XVII sq.) :

Le degré inférieur des liseurs de romans serait représenté par le simple lecteur, d'où il faut bien partir [...]. Le lecteur de romans, c'est celui qui, en fait de romans, lit n'importe quoi, au hasard, sans être guidé par aucun des éléments, intérieurs ou extérieurs, qui tiennent et circulent dans ce mot: le goût. Quand je parlais du plus bas degré, ma phrase suivait la forme, la filière d'une vieille définition philosophique, par Descartes, qui appelle la liberté d'indifférence le plus bas degré de la liberté. Et il y a aussi, chez le lecteur peu lettré qui lit des romans, une sorte d'indifférence qui lui fait accepter n'importe quoi où il y ait de l'aventure, du mouvement, et surtout de la convention. À la base de notre pyramide, voyez cette 
masse énorme, passive et amorphe, de lecteurs de romans. [...] Joignons aux lecteurs de feuilletons les lecteurs de ces romans à bon marché, comme on en voit dans le métro aux mains des ouvrières, et dont il se fait une consommation énorme. les mêmes intentions et selon les mêmes procédés, chez Michel Picard (1986). C'est ainsi
que l'on retrouve, 60 ans après Thibaudet, les mêmes propos : la « masse énorme [...] de
lecteurs de romans » d'Albert Thibaudet deviendra le « peuple » de Michel Picard (cf. la citation reproduite plus haut) ou son " public populaire » ou ses «proliférantes » [sic] et nouvelles «classes moyennes» (p. 267). Albert Thibaudet voit dans les «ouvrières » les représentantes typiques du « lecteur de romans » qui, « en fait de romans, lit n'importe quoi »; pour Picard, c'est une « employée des PTT » qui illustrera ce fait : « Elle lit tout et n'importe quoi »... Les mots mêmes sont repris : la « consommation » de Thibaudet est un leitmotive de Michel Picard, la «masse » des lecteurs de romans vue comme «passive et amorphe » (Thibaudet) se retrouve dans la «passivité amorphe» de Michel Picard (p. 309); et ce qui distingue l'époque de Thibaudet et celle de Picard, c'est que le premier peut tranquillement revendiquer «le goût », quand Michel Picard croit devoir déplorer que «la sociologie et l'histoire ont relativisé le bon goût» (p. 238)... Quant aux dichotomies vues plus haut, on les retrouve évidemment : le liseur s'oppose au lecteur chez Albert Thibaudet comme le lecteur « déchiffreur » et «lecteur» (p. 301 et 306) ou à l'« anti-lecteur » (p. 252). Et, bien sûr, chez Michel Picard, comme chez Albert Thibaudet, c'est que cette dernière catégorie représente « l'immense majorité des lecteurs » (p. 253).

Mais le plus grave évidemment dans ce discours est la conclusion qui en est tirée : par sa référence à Descartes qui, dit-il, « appelle la liberté d'indifférence le plus bas degré de la liberté ", Albert Thibaudet en arrive à définir, à partir de sa modélisation de la lecture, deux degrés de liberté des personnes ainsi catégorisées. C'est précisément la conclusion de l'essai de Michel Picard: pour lui, la lecture littéraire, «si importante pour l'être humain » (p. 307), par sa « fonction modélisante », «produit [...] un nouveau citoyen » (p. 264). Autrement dit, la hiérarchie sociale serait ici justifiée par une telle conception de la lecture.

Même discours, entre ces deux auteurs, chez Jean Guéhenno : dix ans après la fin de la guerre, il établit les mêmes liens entre culture classique et liberté : « Toutes les faiblesses n'avaient été que l'effet de l'ignorance. Il ne fallait qu'augmenter la conscience du peuple, il ne fallait que plus de lumières » (p. 20). Se dessine alors le lien entre culture classique et liberté d'un côté, entre bêtise et asservissement de l'autre : «Il y a dix ans, écrit-il, au sortir de la bêtise et de la honte [...] nous ne voyions plus de limites à ce que pourrait devenir la culture et la liberté des Français» (p. 20). La France, on l'a vu, est alphabétisée ? Il lui manque d'accéder cette fois à la « vraie liberté », à laquelle on accède par la « vraie culture » (p. 22).

Alain Bentolila a pour sa part proposé des variations sur ce discours, à propos de l'illettrisme, pour construire la figure de l'illettré comme non citoyen, voire non humain (l'illettré étant comparé au bonobo)... Bernard Lahire a précisément décrit la structure du discours social actuel sur l'illettrisme, qu'il caractérise ainsi (1999: 16) : 
Le seul rapport entre, d'une part, les pratiques réelles, quotidiennes de l'écrit (lu ou produit) et les rapports effectifs à ces multiples pratiques et, d'autre part, les discours publics sur l'« illettrisme », est un rapport ambigu, opaque et, pourrait-on dire, relativement arbitraire. Tout se passe comme si la même réalité pouvait donner lieu à des découpages, des mises en forme symboliques-politiques de factures différentes. [...] Il n'y a ainsi aucun rapport de transparence entre les discours et la réalité dont ils prétendent parler. inalement, même si les intentions sont différentes, certaines approches didactiques qui font de la distance une caractéristique première de la lecture littéraire, quand elles ne se fondent pas sur une analyse de pratiques sociales effectives et sur une réflexion quant à leur intégration possible dans les classes (Daunay, 2002). Aussi l'urgence me parait-elle, dans l'approche scolaire de la littérature, de l'école au lycée, de se départir d'une conception lettrée (parfois cachée) de la lecture et d'interroger à la fois les représentations des enseignants et des élèves, les pratiques sociales réelles des élèves comme des enseignants et surtout le lien entre les pratiques des élèves et les pratiques scolaires: si un tel projet didactique n'est pas nouveau, force est de constater qu'il est encore minoritaire.

\section{BIBLIOGRAPHIE}

ACADÉMIE FRANÇAISE (2000) : Déclaration de l'Académie française sur le recul des études littéraires, 6 avril 2000, Site de l'académie : <http://www.academie-francaise.fr/actualites/index.html> AGATHON [pseudonyme pour Henri Massis et Alfred de Tarde] (1911) : L'Esprit de la nouvelle Sorbonne. La crise de la culture classique, la crise du français, Paris, Mercure de France. ANGENOT, M. (1982) : La Parole pamphlétaire. Typologie des discours modernes, Paris, Payot. BALIBAR, R. (1974) : Les Français fictifs. Le rapport des styles littéraires au français national, Paris, Hachette.

BARREAU, J.-M. (2005) : L'école et les tentations réactionnaires, Paris, L'Aube.

BEAUNIER, A. (1912) : Pour la Défense française. Les plus détestables bonshommes, Paris, Plon. 
BOUTAN, P. (2000) : Ferdinand Brunot. Crise du français et nomenclature grammaticale officielle (1905-1911), ÉLA, 118, 179-195.

BOUTAN, P. et SAVATovsKY, D. (2000) : Avant-propos, ÉLA, 118, 133-143.

BRUNOT, F. (1911) : La crise du français, Revue hebdomadaire, 14 janvier, 160-176 et 21 janvier, 304-326.

COLLECTIF (2000) : C'est la littérature qu'on assassine rue de Grenelle, Le Monde, 4 mars 2000. DAUNAY, B. (2002) : Éloge de la paraphrase, Saint-Denis, Presses Universitaire de Vincennes. DAUNAY, B. (2003) : Les discours sur l'écriture d'invention et les frontières de la discipline, Recherches, 39, 39-68.

DAUNAY, B. (2004) : L'infini processus de disqualification du lecteur ou contre une didactique bathmologique, in A. Rouxel et G. Langlade, dir., Le sujet lecteur. Lecture subjective et enseignement de la littérature, Rennes, Presses universitaires de Rennes, 233-243.

ÉTIENNE, S. (1933/1965) : Défense de la philologie et autres écrits, Bruxelles, La Renaissance du livre.

FINKIELKRAUT, A. (1987) : La Défaite de la pensée, Paris, Gallimard.

FINKIELKRAUT, A. (2000) : La révolution cuculturelle à l'école, in M. Jarrety, dir., 91-96.

GUÉHENNO, J. (1954) : Lire, Cahiers des bibliothèques de France, 2, 21- 26.

GUENEAU, L. (1922) : Le recrutement des lycées, Revue universitaire, 1922-1, 163-164.

ISAMBERT JAMATI, V. (1985) : Les primaires, ces « incapables prétentieux », RFP, 73, 57-65.

JARRETY, M. (dir) (2000) : Propositions pour les enseignements littéraires, Paris, PUF.

JARRETY, M. (2000) : La nostalgie de l'ambition, in M. Jarrety, dir., 3-11.

JARRETY, M. (2001) : L'avenir d'un passé, Europe, 863, 199-206.

JEY, M. (1998) : La littérature au lycée. Invention d'une discipline, Metz, Faculté des Lettres et Sciences humaines.

LAHIRE, B. (1999) : L'Invention de l'illettrisme, Paris, La Découverte.

LAHIRE, B. (2004) : La culture des individus. Dissonances culturelles et distinction de soi, Paris, La Découverte

LANSON, G. (1903) : Les études modernes et l'enseignement secondaire, in L'Éducation de la démocratie. Leçons professées à l'école des Hautes études sociales, Paris, Félix Alcan.

LANSON, G. (1905) : L'enseignement secondaire, in Enseignement et démocratie. Leçons professées à l'école des hautes études sociales, Paris, Félix Alcan.

LANSON, G. (1909) : La crise des méthodes dans l'enseignement du français, in L'enseignement du français, Conférences du musée pédagogique, Paris, Imprimerie Nationale, 1-24.

LANSON, G. (1925) : Méthodes de l'histoire littéraire, Paris, Les Belles Lettres.

LECHERBonNiER, B. (2005) : Pourquoi veulent-ils tuer le français?, Paris, Albin Michel.

PICARD, M. (1986) : La lecture comme jeu, Paris, Minuit.

PRIVAT, J.-M. et VINSON M.-C. (1999) : Le statut du livre et du lecteur dans les instructions officielles du primaire et du secondaire, Pratiques, 101-102, 105-115.

THIBAUDET, A. (1925) : Le Liseur de romans, Paris, G. Crès et Cie. 
ZINK, M. (2000) : La littérature pour elle-même, in M. Jarrety, dir., 51-55.

SAUVER LES LETTRES (2001) : Des professeurs accusent, Paris, Textuel.

\section{NOTES}

1. Rhétorique souvent décortiquée depuis longtemps... Cf. encore, récemment, Barreau (2005), qui traite des discours « réactionnaires » sur l'école.

2. Que décrit Angenot (1982: p. 85 sq).

3. L'usage de ces expressions est symptomatique, puisqu'elles renvoient à peu près à l'époque où nait la thématique de l'auteur : entre Julien Benda (dont la pensée a été exploitée par Finkielkraut, avec moins de désinvolture que Lecherbonnier, mais dans un ouvrage dont le titre ne dépare pas la collection des cris d'alarme que le xxe siècle a régulièrement produits : La Défaite de la pensée, en 1987) et la guerre d'Espagne...

4. Le numéro de ÉLA est paru en juin 2000, et les auteurs ne peuvent qu'évoquer dans une note (p. 134) notre dernière crise-du-français, mais ils la situent dans la continuité de ce discours de crise. J'y ferai allusion rapidement dans la suite de cet article mais je me permets de renvoyer à l'analyse que j'en ai faite ailleurs (Daunay, 2003) sur un aspect particulier : l'écriture d'invention.

5. La formule « crise du français » désigne en effet indifféremment (parfois même confusément) l'une et l'autre. Si Brunot (1911 : 163) fait la distinction, dans sa réponse à un libelle contre la « Juiverie franc-maçonnique » qui s'en prend entre autres à la terminologie grammaticale, il est assez rare que la distinction soit opératoire. Voir Boutan (2000).

6. Que mes recherches sur la paraphrase m'ont amené à voir fonctionner concrètement, sur un aspect particulier (Daunay 2002).

7. Cf. Jey (1998: 212). Le terme revient dans certains pamphlets récents : $c f$. Barreau (2005:62).

8. J'interroge ici la continuité relative, dans des discours tenus à un siècle de distance sur l'enseignement de la littérature, des formes de disqualification sociale des publics. Il faudrait établir le lien entre cette disqualification des publics et celle des enseignants, des formateurs et des tenants des sciences de l'éducation : comme l'avait montré il y a déjà 20 ans Viviane Isambert Jamati (1985), le mépris des « primaires » concerne aussi bien, dans ces discours, ceux qui sont censés pervertir les savoirs, assimilés aux « primaires », ces « incapables prétentieux ».

9. Gustave Lanson l'illustre en observant que, de 50000 élèves qui atteignaient l'enseignement secondaire un siècle plus tôt, on est passé à 170000 ou 180000 élèves... 10. Sur cette thématique de la frontière collège-lycée et sur les discours concernant l'écriture d'invention, $c f$. Daunay (2003).

11. Qui véhiculent une conception du littéraire comme intouchable, sacrée, accessible seulement par l'explication de texte magistrale, le commentaire et la dissertation, bannissant la paraphrase et l'écriture hypertextuelle (Finkielkraut, 2000) : effet de naturalisation de la littérature bien connu, qui nécessite l'économie d'une définition un peu sérieuse de cette dite littérature, si l'on ne considère pas comme telles les incantations romantiques de « Sauver les lettres » et de leurs tuteurs (dont Jarrety et al.). 12. Ce qui redonne d'ailleurs une réelle actualité aux analyses de Balibar (1974)... 
13. Sur les traces de cette coupure dans les instructions officielles d'avant la réforme, $c f$. Privat et Vinson (1999).

14. J'ai développé les analyses succinctes que je fais ci-dessous dans Daunay (2004).

15. Albert Thibaudet lui-même affirmait, avec une certaine humilité : «Quel est le genre d'action de la littérature sur le lecteur, et surtout sur les lectrices, puisque plus des trois quarts de son public sont un public féminin ? Je ne sais pas trop. Il faudrait une enquête très longue, très vaste et très bien menée dans les milieux populaires » (p. XIX).

16. La multiplication des descriptions de cette figure laisse d'ailleurs entrevoir son origine romanesque ou essayiste (Lahire, 2004 : 124).

\section{RÉSUMÉS}

Les prises de positions récentes sur la dernière réforme de l'enseignement du français, dernier avatar de la séculaire « crise du français ", font ressortir le caractère discriminatoire de certains propos sur la littérature et sur son enseignement. L'auteur en analyse quelques réalisations, du début du xxe siècle à nos jours, en montrant les disqualifications sociale et scolaire qui les soustendent. Ces discours trouvent parfois leur légitimité dans l'approche théorique de la lecture, par la construction de catégories de lecteurs - pourtant peu étayées empiriquement.

\section{AUTEUR}

\section{BERTRAND DAUNAY}

ÉA Théodile, Université Charles de Gaulle-Lille 3. 